\title{
Active Control of Spike-Timing Dependent Synaptic Plasticity in an Electrosensory System
}

\author{
Patrick D. Roberts and Curtis C. Bell \\ Neurological Sciences Institute, OHSU \\ 505 N.W. $185^{\text {th }}$ Avenue, Beaverton, OR 97006 \\ e-mail: robertpa@ohsu.edu
}

June 20, 2002

\begin{abstract}
A spike timing dependent learning rule is present at the synapse between parallel fibers and Purkinje-like medium ganglion cells in the electrosensory lobe of mormyrid electric fish. The synapse is depressed when a postsynaptic dendritic spike occurs within $50 \mathrm{~ms}$ of the onset of a parallel fiber excitatory postsynaptic potential, but is enhanced at all other timing relations. Operation of this learning rule results in the cancellation of predictable membrane potential changes, driving the cell towards a constant output frequency. But medium ganglion cells show a strong and predictable response to corollary discharge signals associated with the motor command that initiates the electric organ discharge. The modeling study presented here resolves this conflict by proposing an active control of dendritic spike threshold during the brief period of medium ganglion cell response.
\end{abstract}

Keywords: mormyrid, plasticity, modeling, electrosensory

\section{Introduction}

The goal of this project is to understand how rules of synaptic plasticity are modulated in an adaptive cerebellum-like sensory structure, the electrosensory lobe (ELL) of mormyrid electric fish. This study uses mathematical analysis and numerical simulation techniques to quantify properties of inputs to the ELL from electrosensory and central sources [10]. These mathematical methods are then used to predict the unobserved synaptic connections between identified neurons in the ELL.

The ELL is a laminar structure that receives primary afferents from electroreceptors in the skin. The signals from one clas of electroreceptors, the mormyromasts, are very sensitive to differences in electrosensory field strength [1] and respond with 1-3 spikes to each electric organ discharge (EOD). The latency of the spikes in mormyromast afferents following the EOD reflects the intensity of the electric field at the receptor. Weak fields generate a single spike with a latency up to $12 \mathrm{msec}$, and strong fields generate multiple spikes with a latency of at least $2 \mathrm{msec}$. The fish uses this latency information to identify objects in its environment by detecting changes in the EOD evoked electric field caused by external objects. 
However, the representation of the electric field strength as latency (pulse coding) would be highly sensitive to information losses due to noise as the information passes through synaptic junctions. As the electrosensory information passes through the first stage of sensory processing, the deep granule layer of the ELL, the signal converges with inputs from central sources that convey timing information about when the EOD occurred. The convergence of these two inputs is thought to be translated via granule cells that act as coincidence detectors and relay a burst of spikes where the number of spikes depends on the time difference between the EOD and the afferent spike [3]. When the sensory information reaches the principal cells of the ganglion layer, much of the timing code is lost and a rate coding can be identified.

The principal cells are divided into two classes: the Purkinje-like medium ganglion cells $\left(\mathrm{MG}_{1}\right.$ and $\mathrm{MG}_{2}$ ) with axons that remain within the ELL, and two types efferent cells, large fusiform (LF) and large ganglion (LG) cells, that project to other centers for further sensory processing. The MG cells are highly adaptive to changes in electrosensory strength and are thought to provide an adaptive filter mechanism to the system by projecting onto the efferent cells and adjusting their output rate. The mechanism for adaptation appears to be a spike-timing dependent learning rule [4] at the synapse that conveys EOD timing information via parallel fibers to the apical dendrites of MG cells.

In the moromyromast regions of ELL, the MG cells respond to the EOD with a short burst of spikes. The efferent cells are usually spontaneously active with a spike rate modulated by the combination of electrosensory and EOD command timing signals. $\mathrm{MG}_{1}$ and LG cells cells are inhibited by the EOD-evoked activity in mormyromast afferents, while $\mathrm{MG}_{2}$ cells and LF cells are excited by such activity.

We have constructed a mathematical model of a hypothesized ELL module consisting of these four cell types. The neurons are formalized as spike-response models that respond to synaptically applied inputs by changing their probability of generating spikes. Key parameters of the model and the postsynaptic inputs to each cell are based on experimental data; deep layer inputs are based on experiments performed in vivo (C. Mohr and C. Bell, unpublished results), and parallel fiber excitatory postsynaptic potentials (EPSP) and synaptic learning rates are derived form experiments performed in vitro $[4,8]$.

We have used the model to test whether the hypothesized connectivity of the ELL module can explain the response and adaptation characteristics of the component neurons. The model is also used to derive constraints on the possible range of synaptic connections that is consistent with experimentally derived results.

\section{Materials and methods}

The model of the four cell neural network uses a spike-response model [6] with no relative refractory period to represent the individual neurons. Time is discretized so that $t_{n}=n \Delta t$, with $n$ an integer, and $\Delta t=1 \mathrm{msec}$. The probability of a spike during the time interval $t_{n}$ is a threshold (sigmoid) function of the membrane potential, $V\left(t_{n}\right)$. For each neuron $A \in\left\{\mathrm{MG}_{1}, \mathrm{MG}_{2}, \mathrm{LF}, \mathrm{LG}\right\}$ in Fig. 1, with threshold $\theta_{A}$, and noise parameter $\mu_{A}$, the spike probability is given by the expression, $f_{A}\left(t_{n}\right)=\left(1+\exp \left[-\mu_{A}\left(V_{A}\left(t_{n}\right)-\theta_{A}\right)\right]\right)^{-1}$. The instantaneous spike frequency is obtained by multiplying the spike probability by the maximum spike frequency.

The membrane potential is the sum of all external inputs. Excitatory synapses from adaptable inputs are represented by weighted excitatory postsynaptic potential waveforms, $\epsilon\left(t_{n}\right)$. The weighting factors, $w^{A}\left(t_{n}\right)$, change in $t_{n}$ as the system adapts due to a learning rule that depends on the relative 


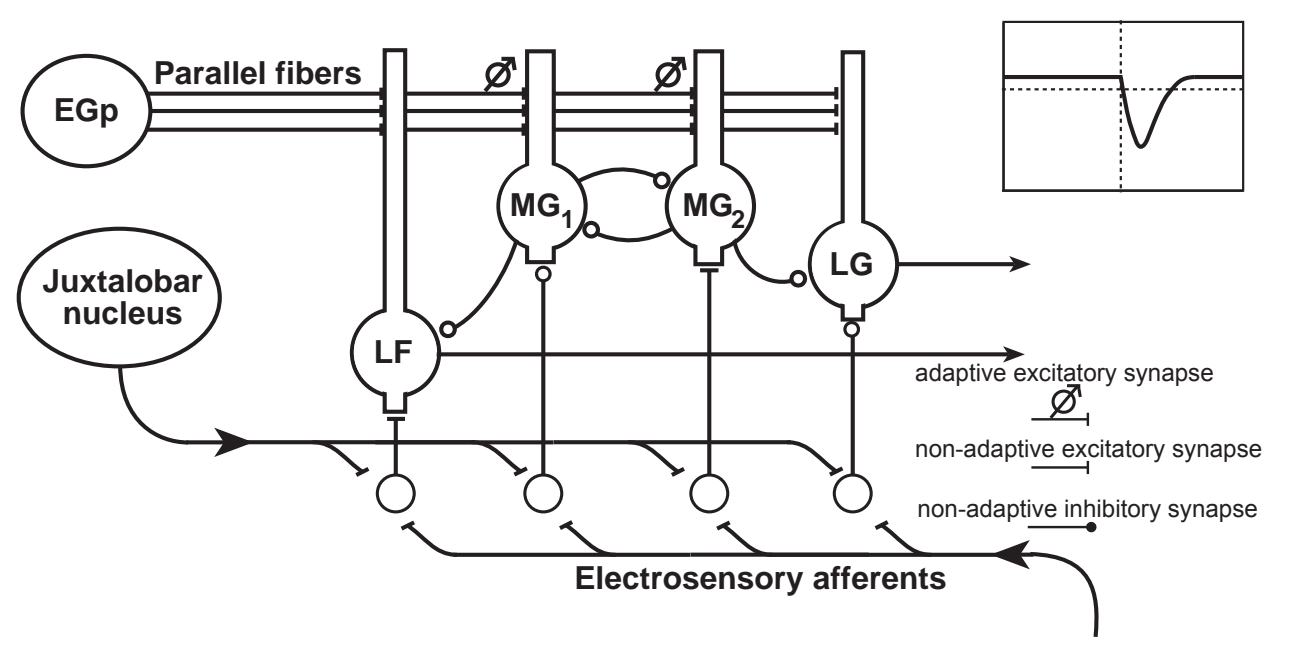

Figure 1: Lateral connectivity between adapting neurons. The model spiking neurons generate spikes that are the postsynaptic events required for associative synaptic plasticity at the adaptive synaptic inputs. Two types of inputs are included in the model, non-adapting inputs to the somas and basal dendrites and adaptive synaptic inputs conveyed by parallel fibers to the apical dendrites. Each adaptive input contributes an excitatory postsynaptic potential (EPSP) to the membrane potential represented by an EPSP waveform weighted by $w_{i}\left(t_{n}\right)$. The membrane potential is the sum of all inputs, adaptive and non-adaptive. Inset: The spike-timing dependency of the learning rule applied to the adaptive synapses. The associative component of the learning rule is the same function as the EPSP waveforms.

timing of pre- and postsynaptic spikes [5]. The contribution of adaptable inputs to the neuron's membrane potential is given by, $V_{A}\left(t_{n}\right)=\sum_{m} w_{m}^{A}\left(t_{n}\right) \epsilon\left(t_{n}-t_{m}\right)$. The non-adaptive inputs are represented by the term $\nu_{A}\left(t_{n}\right)$. The contribution from another neuron $B$ is represented by the spike probability convolved with the postsynaptic potential waveform, and weighted with the synaptic strength $s_{A B}$. For excitatory synapses $s_{A B}>0$, and inhibitory synapse are approximated by using the same postsynaptic potential waveform with $s_{A B}<0$. The membrane potential of neuron $A$ is

$$
V_{A}\left(t_{n}\right)=\sum_{m} w_{m}^{A}\left(t_{n}\right) \epsilon\left(t_{n}-t_{m}\right)+\nu_{A}\left(t_{n}\right)+\sum_{B} s_{A B} \sum_{m} f_{B}\left(t_{m}\right) \epsilon\left(t_{m}-t_{n}\right),
$$

where the last term represents the contribution from the activity of neurons that synapse onto $A$ which are themselves affected by $A$ 's firing (recurrence). In the circuits of ELL this reflects the reciprocal connections between $\mathrm{MG}_{1}$ and $\mathrm{MG}_{2}$ cells (Fig. 1).

The spike-timing dependent learning rule has two components, a non-associative part and an associative part [4]. The change in each synaptic weight per presynaptic spike is $\Delta w\left(t_{\text {pre }}\right)=\alpha-$ $\beta L\left(t_{\text {post }}-t_{\text {pre }}\right)$ The parameters $\alpha$ and $\beta$ denote the non-associative and associative learning rates. The learning function, $L\left(t_{\text {post }}-t_{\text {pre }}\right)$, is positive definite and has unit area.

The learning dynamics are investigated by calculating the ensemble average of the synaptic weights using the spike probability function, $f_{A}\left(t_{n}\right)$,

$$
<\Delta w\left(t_{\text {pre }}\right)>=\alpha-\beta \sum_{n} L\left(t_{n}-t_{\text {pre }}\right) f_{A}\left(t_{n}\right) .
$$

For a single neuron (no recurrence) this expression can be solved near the asymptotic value of the probability function [9], $f_{A}\left(t_{n}\right) \rightarrow \hat{f}=f_{A}(\hat{V})$ as $t_{n} \rightarrow \infty$, where $\hat{f}=\alpha / \beta$. The spike probability approaches its asymptotic value with an exponential decay that can be calculated analytically 
[10]. The real part of the decay constant, $\tau$, is given by $\operatorname{Re}(1 / \tau)=(1 / 2) \beta \mu \hat{f}(1-\hat{f})[10]$. Thus, the asymptotic value and the decay constant depend on the adaptive input rate and parameters of the learning rule. These formula have been implemented in custom simulation software. Further details about the mathematical methods and the simulation code can be found on-line at: http://www.ohsu.edu/nsi/faculty/robertpa/lab.

\section{Results}

In the following we demonstrate the learning dynamics that result from the spike-timing dependent synaptic learning rule [4], and how these dynamics affect the responses to command signals. Then, we show how the original model must be modified to explain the response observed in light of the dynamics.

The basic structure of the ELL is assumed to be grouped into modules that have like the one illustrated in Fig. 1. Three electric organ discharge related inputs act on the modules. One of these is the response of mormyromast afferents to the current generated by the EOD itself. The other two are corollary discharge signals from other central structures associated with the motor command that initiates the EOD. One of these corollary discharge signals arises from the juxtalobar nucleus and terminates in the deep layers of ELL (Fig. 1). The juxtalobar input to granule cells provides the timing signal necessary for measuring latency of afferent responses. A second type of corollary discharge signals arises from EGp and is conveyed as parallel fibers to the molecular layer of ELL Different parallel fibers are presumed to fire at different delays following the EOD motor command.

Focusing on the medium ganglion cells, the parallel fibers show plasticity that is characterized by depression of the synaptic strength when the postsynaptic spike follows the presynaptic spike within a narrow timing window [4]. In vitro experiments showed that the parallel fiber-evoked EPSP is reduced if a postsynaptic dendritic (broad) spike follows the parallel fiber stimulus within a short delay. The EPSP is enhanced if the dendritic spike arrives at other timings with respect to the parallel fiber stimulus (inset in Fig. 1). The learning dynamics of this particular spike-timing dependent learning rule are well understood and lead to the cancellation of input signals that are temporally correlated with the parallel fiber delay series [10].

Our model of the medium ganglion cells consists of a threshold device with noise that generates two types of spikes, a dendritic and an axon spike, when the membrane potential plus noise crosses a threshold. The threshold of the dendritic spike is higher than that of the axon spike, as shown experimentally [7]. In Fig. 2A, the two lines represent the membrane potential, $V_{M G_{1}}\left(t_{n}\right)$ and $V_{M G_{2}}\left(t_{n}\right)$, during the first $200 \mathrm{msec}$ following the command signal alone, and the dashed lines are the dendritic spike thresholds, $\theta_{M G_{1}}\left(t_{n}\right)$ and $\theta_{M G_{2}}\left(t_{n}\right)$. The synaptic strengths are represented by the squares where the initiation time of each excitatory postsynaptic potential is shown and its elevation above the baseline represents the weight of that EPSP.

If we initiate this simulation with the weights at a low value, the weights increase due to the nonassociative component of the learning rule, the membrane potential increase since there no dendritic spikes to depress the EPSPs. The membrane potential reaches a level where the dendritic spikes begin to appear. When the dendritic spike activity reaches an asymptotic value, $\hat{f}=\alpha / \beta$, the progression of the learning stops and the neurons maintain a constant level of output, Fig. $2 \mathrm{~B}$.

If the synaptic weights are initiated at a higher level so that many dendritic spikes are generated, the dendritic spikes depress the parallel fiber evoked EPSPs that contribute to the membrane potential until it reaches that level where the non-associative enhancement and the associative depression 
exactly cancel.

Due to the adaptation of neural responses to the command in the ELL, the system cancels predictable imputs $[1,10]$. Any input that is correlated with the timing of the parallel fiber evoked EPSPs, and therefore correlated with the EOD command, will be cancelled by this mechanism. This simulation starts with the strong depolarization at the beginning of the cycle. The high probability of dendritic spikes caused by the depolarization lead to a depression of the synaptic efficacy until the membrane potential flattens out. Although this mechanism of sensory image cancellation successfully explains the operation of medium ganglion cells in the region of the ELL that receives ampullary afferents, this leads to a paradox when we consider medium ganglion cells that receive their sensory inputs from mormyromast receptors in another region of the ELL.

Medium ganglion cells respond to the EOD motor command with an alteration in membrane potential that is predominately excitatory [2]. This excitation is probably due to juxtalobar input to MG cells. This response to the command is not cancelled by adaptive processes. In light of the learning dynamics, this lack of cancellation is paradoxical. The model cannot explain how medium ganglion cells consistently respond to the command with a burst of spikes. Input correlated with the motor command should be cancelled by plasticity at parallel fiber synapses.

One possible mechanism for the persistent excitatory response of the medium ganglion cells to the command input from the juxtalobar nucleus is that this input not only elicits an EPSP in medium ganglion cells but also elicits a transient elevation in the threshold of the dendritic spike. Such a threshold elevation would modulate the spike-timing dependent learning rule of synaptic plasticity following the command, allowing the system to adapt toward a certain level of excitation following each command.

There is experimental evidence for this kind of modulation of the dendritic spike threshold (C. Mohr and C. Bell, unpublished). In recordings of medium ganglion cell responses to the command, a pause in dendritic spike activity is observable during a brief period that coincides with the command driven EPSP. An increased dendritic spike threshold during the same period is also indicated directly by an increase in the amount of intracellular current necessary to evoke a dendritic spike during the same period.

We have inserted the predicted and experimentally confirmed command driven increase in the dendritic spike threshold into our model neurons (Fig. 2C). Now when we run the simulation the command driven EPSP is still present. This system is now stable under the spike-timing dependent learning rule and is consistent with the experimentally demonstrated command driven EPSP.

\section{Discussion}

Introducing modifications into our model of ELL generates closer agreement with experimental evidence. The modification of the model also gives us a deeper understanding of how adaptable synaptic processes may be controlled. This mathematical model and simulation demonstrate the use of modeling to study the intrinsic learning dynamics of a complex system. Knowledge of these dynamics have been used to make predictions about how the system will operate. When these predictions were in conflict with in vivo recordings, we hypothesized new regulatory mechanisms in this system. Spike timing dependent synaptic plasticity has been observed in other systems (for review see [11]). Modulation of such plasticity by modulating the threshold of a dendritic spike could be an operative mechanism in these other systems as well. 


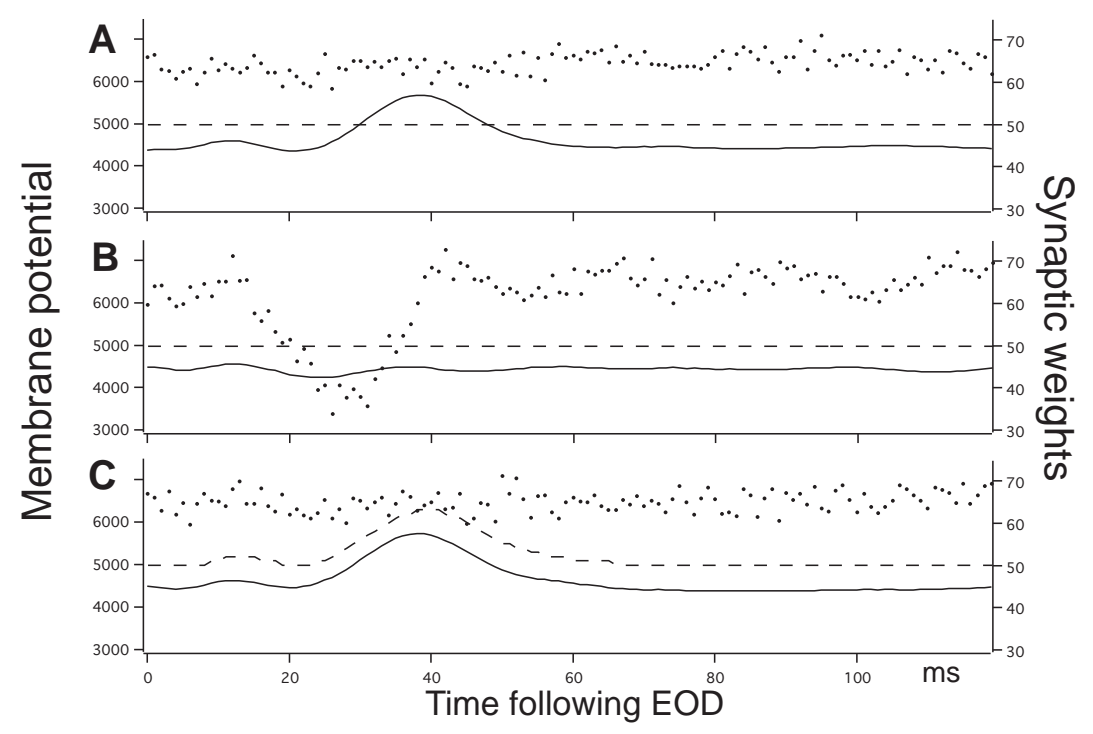

Figure 2: Simulation Results. Membrane potential (solid line), dendritic spike threshold (dashed line), and synaptic weights (separate dots) for a model MG cells. (A) The idealized waveform representation stimulation of the juxtalobar nucleus with the parallel fiber synaptic strengths given a random distribution (arbitrary units). The horozontal location of the weights represents the delay following the EOD command that their input begins, and the height represents the synaptic strength. (B) After adaptation has adjusted the synaptic weights. The synaptic weights for parallel fibers onto the model MG cell are depressed resulting in a cancellation of the juxtalobar evoked EPSP. The cancellation of the juxtalobars nucleus stimulus waveform is absorbed in the profile of the weights. (C) The modified model in which the dendritic spike threshold is increases during the time course of the juxtalobar-evoked EPSP, allowing this waveform to persist in the presence of parallel fiber synaptic plasticity. Noise parameter $\mu=0.01$. 


\section{Acknowledgments}

We would like to thank Drs. Claudia Mohr and Victor Han for discussions and support. This research is supported in part by a National Science Foundation grants IBN 98-08887 (awarded to PDR), and by National Institutes of Health grants R01-MH49792 (awarded to CCB), R01-MH60996 (awarded to CCB), and R01-MH60996 (awarded to PDR).

\section{References}

[1] Curtis C. Bell. Sensory coding and corollary discharge effects in mormyrid electric fish. J. Exp. Biol., 146:229-253, 1989.

[2] Curtis C. Bell, Angel Caputi, and Kirsty Grant. Physiology and plasticity of morphologically identified cells in the mormyrid electrosensory lobe. J. Neurosci., 17:6409-6423, 1997.

[3] Curtis C. Bell and Kirsty Grant. Sensory processing and corollary discharge effects in the mormyromast regions of the mormyrid electrosensory lobe: II. Cell types and corollary discharge plasticity. J. Neurophysiol., 68:859-875, 1992.

[4] Curtis C. Bell, Victor Han, Yoshiko Sugawara, and Kirsty Grant. Synaptic plasticity in a cerebellum-like structure depends on temporal order. Nature, 387:278-281, 1997.

[5] Wulfram Gerstner, Richard Kempter, J. Leo van Hemmen, and Hermann Wagner. A neuronal learning rule for sub-millisecond temporal coding. Nature, 383:76-78, 1996.

[6] Wulfram Gerstner and J. Leo van Hemmen. Associative memory in a network of 'spiking' neurons. Network, 3:139-164, 1992.

[7] Kirsty Grant, Yoshiko Sugawara, Lionel Gomes, Victor Han, and Curtis C. Bell. The Mormyrid electrosensory lobe in vitro: Physiology and pharmacology of cells and circuits. J. Neurosci., 18:6009-6025, 1998.

[8] Victor Han, Kirsty Grant, and Curtis C. Bell. Reversible associative depression and nonassociative potentiation at a parallel fiber synapse. Neuron, 27:611622, 2000.

[9] Patrick D. Roberts. Dynamics of temporal learning rules. Phys. Rev. E, 62:4077-4082, 2000.

[10] Patrick D. Roberts and Curtis C. Bell. Computational consequences of temporally asymmetric learning rules: II. Sensory image cancellation. J. Compu. Neurosci., 9:67-83, 2000.

[11] Patrick D. Roberts and Curtis C. Bell. Spike timing dependent synaptic plasticity in biological systems. (to appear in J. Physiol. (Paris)), 2002. 